\title{
Homoclinic Orbits for a Class of Nonperiodic Hamiltonian Systems
}

\author{
Wenping Qin, Jian Zhang, and Fukun Zhao \\ Department of Mathematics, Yunnan Normal University, Yunnan, Kunming 650092, China \\ Correspondence should be addressed to Fukun Zhao, fukunzhao@163.com \\ Received 6 December 2011; Accepted 16 January 2012 \\ Academic Editor: Svatoslav Staněk
}

Copyright (C) 2012 Wenping Qin et al. This is an open access article distributed under the Creative Commons Attribution License, which permits unrestricted use, distribution, and reproduction in any medium, provided the original work is properly cited.

We study the following nonperiodic Hamiltonian system $\dot{z}=2 H_{z}(t, z)$, where $H \in C^{1}\left(\mathbb{R} \times \mathbb{R}^{2 N}, \mathbb{R}\right)$ is the form $H(t, z)=(1 / 2) B(t) z \cdot z+R(t, z)$. We introduce a new assumption on $B(t)$ and prove that the corresponding Hamiltonian operator has only point spectrum. Moreover, by applying a generalized linking theorem for strongly indefinite functionals, we establish the existence of homoclinic orbits for asymptotically quadratic nonlinearity as well as the existence of infinitely many homoclinic orbits for superquadratic nonlinearity.

\section{Introduction and Main Results}

In this paper, we are interested in the existence of homoclinic orbits of the Hamiltonian system

$$
\dot{z}=2 H_{z}(t, z)
$$

where $z=(p, q) \in \mathbb{R}^{N} \times \mathbb{R}^{N}=\mathbb{R}^{2 N}, \partial=\left(\begin{array}{cc}0 & I_{N} \\ -I_{N} & 0\end{array}\right)$, and $H \in C^{1}\left(\mathbb{R} \times \mathbb{R}^{2 N}, \mathbb{R}\right)$ is the form

$$
H(t, z)=\frac{1}{2} B(t) z \cdot z+R(t, z)
$$

with $B(t) \in C\left(\mathbb{R}, \mathbb{R}^{4 N^{2}}\right)$ being a $2 N \times 2 N$ symmetric matrix valued function, and $R \in C^{1}(\mathbb{R} \times$ $\left.\mathbb{R}^{2 N}, \mathbb{R}\right)$. Here, by a homoclinic orbit of (HS), we mean a solution of the equation satisfying $z(t) \not \equiv 0$ and $z(t) \rightarrow 0$ as $|t| \rightarrow \infty$.

Establishing the existence of homoclinic orbits for system like (HS) is one of the most important problems in the theory of Hamiltonian systems. In very recent years, many 
authors devoted to the existences of homoclinic orbits for Hamiltonian systems via critical point theory. For example, see [1-5] for the second-order systems and [6-18] for the firstorder systems. Coti-Zelati et al. first considered the system (HS) in [6], and they obtained a homoclinic orbit for strictly convex Hamiltonian system. The existence of infinitely many homoclinic orbits was established in [15]. Subsequently, Hofer and Wysocki removed the convexity assumptions in [13]. Later, suppose that $R(t, z)$ and $B(t)$ depend periodically on $t$, the existence of homoclinic orbit for (HS) was considered in $[7,8,12,16,18]$.

Without assumption of periodicity, the problem is quite different in nature. In [10], Ding and Li first obtained one homoclinic orbit for the nonperiodic system (HS), see also $[9,11]$ and the references therein for recent works on this direction.

Motivated by $[9,11]$, in this paper, we introduce a new nonperiodic assumption on $B(t)$ as the following.

$\left(\mathrm{B}_{0}\right) B(t) \in C\left(\mathbb{R}, \mathbb{R}^{2 N \times 2 N}\right)$, there exists $r_{0}>0$ such that, for any $h>0$,

$$
\left|\left\{t \in \mathbb{R}:\left|t-t_{1}\right| \leq r_{0}, \partial_{0} B(t)<h\right\}\right| \longrightarrow 0, \quad \text { as }\left|t_{1}\right| \longrightarrow \infty,
$$

where $\partial_{0}$ will be be defined in Section 2; we regard a real function $U(x)$ as a symmetric matrix $U(x) I_{2 N \times 2 N}$, and, for two given matrix valued functions $L_{1}(t)$ and $L_{2}(t)$, we say that $L_{1}(t) \leq L_{2}(t)$ if and only if

$$
\max _{\xi \in \mathbb{R}^{2 N},|\xi|=1}\left(L_{1}(t)-L_{2}(t)\right) \xi \cdot \xi \leq 0,
$$

and $L_{1}(t)>L_{2}(t)$ if and only if $L_{1}(t) \leq L_{2}(t)$ does not hold. Obviously, $\left(\mathrm{B}_{0}\right)$ holds if $B(t)$ satisfies.

$\left(B_{1}\right)$ For any $b>0$, the set $\Lambda^{b}:=\left\{t \in \mathbb{R}: \partial_{0} B(t)<b\right\}$ is nonempty and has finite Lebesgue measure.

One of the main ingredients of our work is two steps, the first is to show that the spectrum of Hamiltonian operator

$$
A:=-\left(2 \frac{d}{d t}+B\right)
$$

consists of a sequence of eigenvalues with finite multiplicity which is unbounded from above and below (see Lemma 2.2), and hence the energy functional corresponding to (HS) is strongly indefinite; the second is to show that the working space $E$ has compact embedding property (see Lemma 2.3).

First, we handle the asymptotically quadratic case. In what follows, $\widehat{R}(t, z):=$ $(1 / 2) R_{z}(t, z) z-R(t, z)$. Suppose that

$\left(\mathrm{R}_{1}\right) R \in C^{1}\left(\mathbb{R} \times \mathbb{R}^{2 N},[0, \infty)\right)$ and $R_{z}(t, z)=o(|z|)$ as $|z| \rightarrow 0$ uniformly in $t$,

$\left(\mathrm{R}_{2}\right)$ there exists a bounded function $R_{\infty} \in C(\mathbb{R}, \mathbb{R})$ such that $\left|R_{z}(t, z)-R_{\infty}(t) z\right| /|z| \rightarrow 0$ as $|z| \rightarrow \infty$ uniformly in $t$, and $b:=\inf _{t \in \mathbb{R}} R_{\infty}(t)>\inf [(0, \infty) \cap \sigma(A)]$, where $\sigma(A)$ denotes the spectrum of $A$,

$\left(\mathrm{R}_{3}\right) \widehat{R}(t, z)>0$ if $z \neq 0, \widehat{R}(t, z) \rightarrow \infty$ as $|z| \rightarrow \infty$. 
We recall that a solution $z_{1}$ of (HS) is called a least energy solution if it possesses minimum energy among all solutions, that is,

$$
\Phi\left(z_{1}\right)=\theta:=\inf \{\Phi(z) \mid z \text { is a nonzero solution of (HS) }\}
$$

where $\Phi$ is the energy functional

$$
\Phi(z):=-\int_{\mathbb{R}}\left(\frac{1}{2} \partial \dot{z} \cdot z+H(t, z)\right) d t
$$

Let $k$ be the number of eigenvalues of the operator $A$ lying in $(0, b)$, our main results are the following.

Theorem 1.1. Suppose $\left(B_{0}\right),\left(R_{1}\right)-\left(R_{3}\right)$ be satisfied. Then, $(\mathrm{HS})$ has a least energy solution. Moreover, if $R(t, z)$ is even in $z$, then (HS) has at least $k$ pairs of solutions.

Next, we consider the superquadratic case. Assume

$\left(\mathrm{R}_{4}\right) R(t, z) \cdot|z|^{-2} \rightarrow \infty$ as $|z| \rightarrow \infty$ uniformly in $t$,

$\left(\mathrm{R}_{5}\right) \widehat{R}(t, z)>0$ if $z \neq 0$, and there are $\mu>1$ and $r, c_{1}>0$, such that, for $|z| \geq r,\left|R_{z}(t, z)\right|^{\mu} \leq$ $c_{1} \widehat{R}(t, z)|z|^{\mu}$.

Theorem 1.2. Suppose $\left(B_{0}\right),\left(R_{1}\right)$, and $\left(R_{4}\right)-\left(R_{5}\right)$ be satisfied. Then, $(\mathrm{HS})$ has a least energy solution. Moreover, if $R(t, z)$ is even in $u$. Then, (HS) has infinitely many solutions.

Remark 1.3. The assumptions $\left(\mathrm{R}_{1}\right),\left(\mathrm{R}_{4}\right)-\left(\mathrm{R}_{5}\right)$ imply that $\widehat{R}(t, z) \rightarrow \infty$ uniformly in $t$ as $|z| \rightarrow$ $\infty$.

\section{Variational Framework and Linking Structure}

In order to establish a variational setting for the system (HS), in this section, we first study the spectrum of the Hamiltonian operator.

Observe that, since we have assumed $\left(\mathrm{B}_{0}\right)$ about $B(t), A=-(2(d / d t)+B(t))$ is a self-dajoint operator on $L^{2}\left(\mathbb{R}, \mathbb{R}^{2 N}\right)$ with $\Phi(A) \subset H^{1}\left(\mathbb{R}, \mathbb{R}^{2 N}\right)$. Let $|\cdot|_{q}$ denote the usual $L^{q_{-}}$ norm, $(\cdot, \cdot)_{2}$ denote the usual $L^{2}$ inner product, $c, c_{i}, d$, or $d_{i}$ stand for different positive constants. Let $\sigma(A), \sigma_{d}(A)$, and $\sigma_{e}(A)$ be the spectrum of $A$, the discrete spectrum of $A$, and the essential spectrum of $A$, respectively. Observe that $\Phi(A)$ is a Hilbert space with the graph inner product

$$
\left(z_{1}, z_{2}\right)_{A}:=\left(A z_{1}, A z_{2}\right)_{2}+\left(z_{1}, z_{2}\right)_{2}
$$

and the induced norm $\|z\|_{A}:=(z, z)_{A}^{1 / 2}$. 
Set $\partial_{0}:=\left(\begin{array}{cc}0 & I_{N} \\ I_{N} & 0\end{array}\right)$ and $A_{0}:=\partial(d / d t)+\partial_{0}$ (self-adjoint with $\left.\Phi\left(A_{0}\right)=H^{1}\left(\mathbb{R}, \mathbb{R}^{2 N}\right)\right)$; thus, $A_{0}^{2}=-d^{2} / d t^{2}+1$. Let $\left|A_{0}\right|$ denote the absolute value of $A_{0}$, and we have

$$
\| A_{0}|z|_{2}^{2}=\left|A_{0} z\right|_{2}^{2}=\left(A_{0} z, A_{0} z\right)_{2}=\left(A_{0}^{2} z, z\right)=\left(\left(-\frac{d^{2}}{d t^{2}}+1\right) z, z\right)_{2}=|\nabla z|_{2}^{2}+|z|_{2}^{2}
$$

which implies that

$$
\|z\|_{H^{1}}=\| A_{0}|z|_{2}
$$

for all $z \in \Phi\left(A_{0}^{2}\right)=H^{2}\left(\mathbb{R}, \mathbb{R}^{2 N}\right)$, hence for all $z \in H^{1}\left(\mathbb{R}, \mathbb{R}^{2 N}\right)$ because of the density of $H^{2}$ in $H^{1}$.

Lemma 2.1. For any $z \in \Phi(A) \subset H^{1}\left(\mathbb{R}, \mathbb{R}^{2 N}\right)$, there exists $d>0$ such that

$$
\|z\|_{H^{1}}=\left\|A_{0}|z|_{2} \leq d\right\| z \|_{A} .
$$

Proof. Let $A_{1}$ be the restriction of $A_{0}$ to $\Phi(A), A_{1}$ is a linear operator from $\Phi(A)$ to $L^{2}$. We claim that $A_{1}$ is closed. Indeed, let $z_{n} \stackrel{\|\cdot\|_{A}}{\longrightarrow} z$ and $A_{1} z_{n} \stackrel{|\cdot|_{2}}{\longrightarrow} w$. Then, $z \in \Phi(A)$, and since $A_{0}$ is closed, $A_{1} z_{n}=A_{0} z_{n} \stackrel{\nvdash_{2}}{\longrightarrow} A_{0} z=A_{1} z$, hence the claim holds. Now the Closed Graph Theorem implies that $A_{1}: \Phi(A) \rightarrow L^{2}$ is a bounded linear operator, so $\left|A_{0} z\right|_{2}=\left|A_{1} z\right|_{2} \leq d\|z\|_{A}$ for all $z \in \boldsymbol{\Phi}(A)$. This together with (2.3) implies (2.4).

Lemma 2.2. Suppose $\left(B_{0}\right)$ holds. Then $\sigma(A)=\sigma_{d}(A)$.

Proof. Similar to the idea of $[9,19]$, for any $h>0$, if $\left(\mathrm{B}_{0}\right)$ holds, set

$$
\left(\partial_{0} B(t)-h\right)^{+}:= \begin{cases}\partial_{0} B(t)-h, & \text { if } \partial_{0} B(t)-h \geq 0 \\ 0, & \text { if } \partial_{0} B(t)-h<0\end{cases}
$$

and $\left(\partial_{0} B(t)-h\right)^{-}=\left(\partial_{0} B(t)-h\right)-\left(\partial_{0} B(t)-h\right)^{+}$. Thus, $A=A_{2}+\partial_{0}\left(\partial_{0} B(t)-h\right)^{-}$, where

$$
A_{2}=-2 \frac{d}{d t}+\partial_{0}\left(\partial_{0} B(t)-h\right)^{+}+h \partial_{0}
$$


Since $\partial_{0}^{2}=I$ and $\partial_{0} \partial=-\partial \partial_{0}$, we have, for $z \in \Phi(A)$,

$$
\begin{aligned}
\left(A_{2} z, A_{2} z\right)_{2}= & \left|\left(-\partial \frac{d}{d t}+\partial_{0}\left(\partial_{0} B(t)-h\right)^{+}\right) z+h \partial_{0} z\right|_{2}^{2} \\
= & \left|\left(-\partial \frac{d}{d t}+\partial_{0}\left(\partial_{0} B(t)-h\right)^{+}\right) z\right|_{2}^{2}+h^{2}|z|_{2}^{2}+\left(-\partial \dot{z}, h \partial_{0} z\right)_{2}+\left(h \partial_{0} z,-\partial \dot{z}\right)_{2} \\
& +\left(\left(\partial_{0}\left(\partial_{0} B(t)-h\right)^{+}\right) z, h \partial_{0} z\right)_{2}+\left(\left(h \partial_{0} z, \partial_{0}\left(\partial_{0} B(t)-h\right)^{+}\right) z\right)_{2} \\
= & \left|\left(-\partial \frac{d}{d t}+\partial_{0}\left(\partial_{0} B(t)-h\right)^{+}\right) z\right|_{2}^{2}+h^{2}|z|_{2}^{2}+2 h\left(\left(\left(\partial_{0} B(t)-h\right)^{+}\right) z, z\right)_{2} \\
\geq & h^{2}|z|_{2}^{2} .
\end{aligned}
$$

Thus, $\sigma\left(A_{2}\right) \subset \mathbb{R} \backslash(-h, h)$.

We claim that $\sigma_{e}(A) \cap(h, h)=\emptyset$. Assume by contradiction that there is $v \in \sigma_{e}(A)$ with $|v|<h$. Let $\left\{z_{n}\right\} \subset \Phi(A)$ with $\left|z_{n}\right|_{2}=1, z_{n} \rightarrow 0$ in $L^{2}$ and $\left|(A-v) z_{n}\right|_{2} \rightarrow 0$. Moreover, by $\left(\mathrm{B}_{0}\right)$, one can check that the multiplication operator $z \rightarrow \partial_{0}\left(\partial_{0} B(t)-h\right)^{-} z$ is compact. In fact, let $\left\{z_{n}\right\}$ be bounded in $\Phi(A)$, without loss of generality, we may assume $z_{n} \rightarrow 0$ in $\Phi(A)$. Next we show that $\left|\partial_{0}\left(\partial_{0} B(t)-h\right)^{-} z_{n}\right|_{2} \rightarrow 0$ in $L^{2}$. For every $R>0$, define $B_{R}(0)=\{t \in \mathbb{R}:|t|<R\}$ and $B_{R}^{c}=\mathbb{R} \backslash B_{R}(0)$. Let $\left\{t_{i}\right\}$ be a sequence of points in $B_{R}^{c}$ satisfying $B_{R}^{c} \subset \cup_{i=1}^{\infty} B\left(t_{i}, r_{0}\right)$ and such that each point $t$ is contained in at most 2 such balls $B\left(t_{i}, r_{0}\right)$. Let $B:=\left\{t \in B_{R}^{c}: \partial_{0} B(t)<h\right\}$, choose $s \in(1,3)$ and $s^{\prime}=s /(s-1)$. We get

$$
\begin{aligned}
\int_{B_{R}^{c}}\left|\partial_{0}\left(\partial_{0} B(t)-h\right)^{-} z_{n}\right|^{2} & \leq \sum_{i=1}^{\infty} \int_{B\left(t_{i}, r_{0}\right) \cap B}\left|\partial_{0}\left(\partial_{0} B(t)-h\right)^{-} z_{n}\right|^{2} \\
& \leq \sum_{i=1}^{\infty}\left(\int_{B\left(t_{i}, r_{0}\right) \cap B}\left|z_{n}\right|^{2 s}\right)^{1 / s}\left(\int_{B\left(t_{i}, r_{0}\right) \cap B}\left|\partial_{0}\left(\partial_{0} B(t)-h\right)^{-}\right|^{2 s^{\prime}}\right)^{1 / s^{\prime}} \\
& \leq C_{R}^{2 s^{\prime}} \sum_{i=1}^{\infty}\left|B\left(t_{i}, r_{0}\right) \cap B\right|^{1 / s^{\prime}}\left(\int_{B\left(t_{i}, r_{0}\right) \cap B}\left|z_{n}\right|^{2 s}\right)^{1 / s} \\
& \leq 2 C_{R}^{2 s^{\prime}} \varepsilon_{R}\left\|z_{n}\right\|_{A^{\prime}}^{2}
\end{aligned}
$$

where $\varepsilon_{R}=\sup _{i}\left|B\left(t_{i}, r_{0}\right) \cap B\right|^{1 / s^{\prime}}, C_{R}=\sup _{i}\left\|\partial_{0}\left(\partial_{0} B(t)-h\right)^{-}\right\|_{M}$ (for real matrix valued function $\left.Q(x)=\left(q_{j k}(x)\right)_{2 N \times 2 N},\|Q(x)\|_{M}=\max _{1 \leq j \leq 2 N} \sum_{k=1}^{2 N}\left|q_{j k}(x)\right|\right)$.

Here, we used (2.4) of Lemma 2.1 and that $H^{1}$ embeds into $L^{2 s}$. Assumption $\left(\mathrm{B}_{0}\right)$ implies that $\varepsilon_{R} \rightarrow 0$ as $R \rightarrow \infty$; thus,

$$
\int_{B_{R}^{c}}\left|\partial_{0}\left(\partial_{0} B(t)-h\right)^{-} z_{n}\right|^{2} \longrightarrow 0
$$


On the other hand,

$$
\int_{B_{R}(0)}\left|\partial_{0}\left(\partial_{0} B(t)-h\right)^{-} z_{n}\right|^{2} \leq\left(\int_{B_{R}(0)}\left|z_{n}\right|^{2 s}\right)^{1 / s}\left(\int_{B_{R}(0)}\left|\partial_{0}\left(\partial_{0} B(t)-h\right)^{-}\right|^{2 s^{\prime}}\right)^{1 / s^{\prime}} \longrightarrow 0,
$$

since $H^{1} \hookrightarrow L_{\text {loc }}^{2}$ is compact. Thus, $\left|\partial_{0}\left(\partial_{0} B(t)-h\right)^{-} z_{n}\right|_{2} \rightarrow 0$, we have

$$
\begin{aligned}
o(1) & =\left|(A-v) z_{n}\right|_{2}=\left|A_{2} z_{n}-v z_{n}+\partial_{0}\left(\partial_{0} B(t)-h\right)^{-} z_{n}\right|_{2} \\
& \geq\left|A_{2} z_{n}\right|_{2}-|v|-o(1) \\
& \geq h-|v|-o(1),
\end{aligned}
$$

which implies that $0<h-|v| \leq 0$, a contradiction. So $\sigma_{e}(A) \cap(-h, h)=\emptyset$. Since $h>0$ is arbitrary, it follows that $\sigma(A)=\sigma_{d}(A)$.

From Lemma 2.2, we know that the operator $A$ has a sequence of eigenvalues

$$
\cdots \lambda_{-k} \leq \cdots \leq \lambda_{-1} \leq 0<\lambda_{1} \leq \cdots \leq \lambda_{k} \cdots
$$

with $\lambda_{ \pm k} \rightarrow \pm \infty$ as $k \rightarrow \infty$, and corresponding eigenfunctions $\left\{e_{ \pm k}\right\}_{k \in \mathbb{N}}$ form an orthogonal basis in $L^{2}$. Observe that we have an orthogonal decomposition

$$
L^{2}=L^{-} \oplus L^{0} \oplus L^{+}, \quad z=z^{-}+z^{0}+z^{+},
$$

such that $A$ is negative definite on $L^{-}$and positive definite on $L^{+}$and $L^{0}=\operatorname{ker} A$. Let $P^{0}$ : $L^{2} \rightarrow L^{0}$ be the projection. Set $E:=\Phi\left(|A|^{1 / 2}\right)$ be the domain of the self-adjoint operator $|A|^{1 / 2}$ which is a Hilbert space equipped with the inner product

$$
(z, w)=\left(|A|^{1 / 2} z,|A|^{1 / 2} w\right)_{2}+\left(P^{0} z, P^{0} w\right)_{2}
$$

and norm $\|z\|=(z, z)^{1 / 2}$. Let $E^{ \pm}:={\overline{\operatorname{span}\left\{e_{ \pm k}\right\}}}_{k \in \mathbb{N}}, E^{0}=$ ker $A$. Then, $E=E^{-} \oplus E^{0} \oplus E^{+}$is an orthogonal decomposition of $E$.

Similar to [19], on $\boldsymbol{\Phi}(A)$, we introduce an inner product

$$
\langle z, w\rangle_{A}=(A z, A w)_{2}+\left(P^{0} z, P^{0} w\right)_{2}=(|A| z,|A| w)_{2}+\left(P^{0} z, w\right)_{2}
$$

whose induced norm will be denoted by $|z|_{A}$. Since 0 is at most an isolated eigenvalue of finite multiplicity, it is clear that $|\cdot|_{A}$ and $\|\cdot\|_{A}$ are equivalent on $\Phi(A): d_{1}|z|_{A} \leq\|u\|_{A} \leq d_{2}|z|_{A}$, for all $z \in \Phi(A)$. Define

$$
\widehat{A}:=|A|+P^{0} .
$$


Then, $\Phi(A)=\Phi(\widehat{A})$. Noting that $P^{0}|A|=|A| P^{0}=0$, for $z, w \in \Phi(\widehat{A})$, we have

$$
\begin{aligned}
(\widehat{A} z, \widehat{A} w)_{2} & =(|A| z,|A| w)_{2}+\left(P^{0} z,|A| w\right)_{2}+\left(|A| z, P^{0} w\right)_{2}+\left(P^{0} z, P^{0} w\right)_{2} \\
& =(|A| z,|A| w)_{2}+\left(P^{0} z, P^{0} w\right)_{2}=\langle z, w\rangle_{A^{\prime}}
\end{aligned}
$$

hence

$$
|z|_{A}=|\widehat{A} z|_{2}, \quad \forall z \in \Phi(A)
$$

Observe that, for all $z \in \mathscr{\Phi}(A)$ and $w \in \boldsymbol{\Phi}\left(|A|^{1 / 2}\right)$,

$$
\begin{aligned}
\left(\widehat{A}^{1 / 2} z, \widehat{A}^{1 / 2} w\right)_{2} & =(\widehat{A} z, w)_{2}=\left(\left(|A|+P^{0}\right) z, w\right)_{2}=(|A| z, w)_{2}+\left(P^{0} z, w\right)_{2} \\
& =\left(|A|^{1 / 2} z,|A|^{1 / 2} w\right)_{2}+\left(P^{0} z, P^{0} w\right)_{2}=(z, w) .
\end{aligned}
$$

Consequently, since $\Phi(A)=\Phi(\widehat{A})$ is a core of $\widehat{A}^{1 / 2}$, we have

$$
(z, w)=\left(\widehat{A}^{1 / 2} z, \widehat{A}^{1 / 2} w\right)_{2} \quad \forall z, w \in \Phi\left(|A|^{1 / 2}\right)
$$

which implies in particular that

$$
\|z\|=\left|\widehat{A}^{1 / 2} z\right|_{2} \quad \forall z \in E
$$

By complex interpolation theory, we have $H^{1 / 2}=\left[L^{2}, H^{1}\right]_{1 / 2}$ (see Theorem 2.4 .1 [20]). Since $\mathscr{\Phi}\left(\left|A_{0}\right|^{0}\right)=L^{2}$ and $\|z\|_{H^{1}}=\| A_{0}|z|_{2}$, one has

$$
H^{1 / 2}=\left[\Phi\left(\left|A_{0}\right|^{0}\right), \Phi\left(\left|A_{0}\right|\right)\right]_{1 / 2}
$$

with equivalent norms. It then follows from Theorem 1.18 .10 of [20] that

$$
H^{1 / 2}=\left[\Phi\left(\left|A_{0}\right|^{0}\right), \Phi\left(\left|A_{0}\right|\right)\right]_{1 / 2}=\Phi\left(\left|A_{0}\right|^{1 / 2}\right),
$$

hence $\|z\|_{H^{1 / 2}}$ and $\|\left.\left. A_{0}\right|^{1 / 2} z\right|_{2}$ are equivalent norms on $H^{1 / 2}$,

$$
d_{3}\|z\|_{H^{1 / 2}} \leq\left.\left.|| A_{0}\right|^{1 / 2} z\right|_{2} \leq d_{4}\|z\|_{H^{1 / 2}} \quad \forall z \in H^{1 / 2}\left(\mathbb{R}, \mathbb{R}^{2 N}\right)
$$

Lemma 2.3. E embeds continuously into $H^{1 / 2}\left(\mathbb{R}, \mathbb{R}^{2 N}\right)$. Moreover, E embeds compactly into $L^{p}\left(\mathbb{R}, \mathbb{R}^{2 N}\right)$ for all $p \in[2, \infty)$. 
Proof. By (2.4) and (2.18), there exists $d_{5}>0$ such that

$$
\| A_{0}|z|_{2} \leq d_{5}|\widehat{A} z|_{2}=\left|\left(d_{5} \widehat{A}\right) z\right|_{2} \quad \forall z \in \Phi(A)
$$

Thus, $\left(\left|A_{0}\right| z, z\right)_{2} \leq\left(d_{5} \widehat{A} z, z\right)_{2}$ for all $u \in \boldsymbol{\Phi}(A)$ (see Proposition III 8.11 of [21]). This implies

$$
\left.\left.|| A_{0}\right|^{1 / 2} z\right|_{2} ^{2}=\left(\left|A_{0}\right| z, z\right)_{2} \leq\left(d_{5} \widehat{A} z, z\right)_{2}=d_{5}\left|\widehat{A}^{1 / 2} z\right|_{2}^{2}
$$

for all $z \in \Phi(A)$ (see Proposition III 8.12 of [21]). Since $\Phi(A)$ is core of $\widehat{A}^{1 / 2}$, we obtain that $\left.\left.|| A_{0}\right|^{1 / 2} z\right|_{2} ^{2} \leq d_{5}\left|\widehat{A}^{1 / 2} z\right|_{2}^{2}$ for all $z \in E$. This combination with (2.21) shows that

$$
\left.\left.|| A_{0}\right|^{1 / 2} z\right|_{2} ^{2} \leq d_{5}\|z\|^{2} \quad \forall z \in E
$$

which, together with (2.24), implies that

$$
\|z\|_{H^{1 / 2}} \leq d_{6}\|z\| \quad \forall z \in E
$$

This proves that the embedding $E \hookrightarrow H^{1 / 2}\left(\mathbb{R}, \mathbb{R}^{2 N}\right)$ is continuous. In order to show that the embedding $E \hookrightarrow L^{p}\left(\mathbb{R}, \mathbb{R}^{2 N}\right)$ is compact for all $p \in[2, \infty)$, it suffices to prove that $E \hookrightarrow L^{2}$ is compact. Set $L_{k}:=\operatorname{span}\left\{e_{-k}, \ldots, e_{-1}, e_{1}, \ldots, e_{k}\right\}$. Let $P_{k}: E \rightarrow L_{k}$ denote the orthogonal projector. Consider a weakly converging sequence $z_{n} \rightarrow z$ in $E$. Denote $w_{n}=z_{n}-z$ and $K:=\sup _{n}\left\|w_{n}\right\|^{2}$. Given $\varepsilon>0$, we choose $k \in \mathbb{N}$ so that $K / v_{k}<\varepsilon / 2$, where $v_{k}:=\left|\lambda_{-k}\right|+\lambda_{k}$. Since $P_{k} w_{n} \rightarrow 0$ as $n \rightarrow \infty$, then there exists $n_{0} \in \mathbb{N}$ such that $\left\|P_{k} w_{n}\right\|^{2}<\varepsilon / 2$ for all $n \geq n_{0}$. Let $\{E(\tau)\}_{\tau \in \mathbb{R}}$ be the spectral family of $A$. It follows from

$$
\begin{aligned}
\left\|w_{n}\right\|^{2} & \geq\left\|\left(I-P_{k}\right) w_{n}\right\|^{2}=\left(|A|^{1 / 2}\left(I-P_{k}\right) w_{n}|A|^{1 / 2}\left(I-P_{k}\right) w_{n}\right)_{2} \\
& =\int_{-\infty}^{\lambda_{-k}}|\tau| d\left|E(\tau)\left(I-P_{k}\right) w_{n}\right|_{2}^{2}+\int_{\lambda_{k}}^{\infty} \tau d\left|E(\tau)\left(I-P_{k}\right) w_{n}\right|_{2}^{2} \\
& \geq\left(\left|\lambda_{-k}\right|+\lambda_{k}\right)\left|\left(I-P_{k}\right) w_{n}\right|_{2}^{2}
\end{aligned}
$$

that

$$
\left|\left(I-P_{k}\right) w_{n}\right|_{2}^{2} \leq \frac{\left\|w_{n}\right\|^{2}}{\left|\lambda_{-k}\right|+\lambda_{k}}=\frac{\left\|w_{n}\right\|^{2}}{v_{k}}<\frac{\varepsilon}{2}
$$

Then,

$$
\left|w_{n}\right|_{2}^{2}=\left|P_{k} w_{n}\right|_{2}^{2}+\left|\left(I-P_{k}\right) w_{n}\right|_{2}^{2}<\frac{\varepsilon}{2}+\frac{\varepsilon}{2}=\varepsilon
$$

for all $n \geq n_{0}$. This proves that $z_{n} \rightarrow z$ in $L^{2}$. 
Set $\Psi(z)=\int_{\mathbb{R}} R(t, z(t)) d t$, by assumptions and Lemma 2.3, $\Psi(z) \in C^{1}(E, \mathbb{R})$ and $\Psi^{\prime}(z) w=\int_{\mathbb{R}} R_{z}(t, z(t)) w(t) d t$, for all $z, w \in E$. Now, let us consider the function

$$
\Phi(z)=\frac{1}{2}\left(\left\|z^{+}\right\|^{2}-\left\|z^{-}\right\|^{2}\right)-\Psi(z)
$$

for $z=z^{+}+z^{0}+z^{-} \in E$, then $\Phi \in C^{1}(E, \mathbb{R})$. Moreover, for $\Psi \in C_{0}^{\infty}(\mathbb{R})$,

$$
\Phi^{\prime}(z) \Psi=\int_{\mathbb{R}}\left(-\partial \dot{z}(t)+B(t) z(t)-R_{z}(t, z(t)), \Psi(t)\right) d t
$$

It follows that critical points of $\Phi(z)$ are solutions of (HS). Moreover, if $z$ is a solution of (HS), by Lemma 2.3, $R_{z}(t, z) \in L^{s}\left(\mathbb{R}, \mathbb{R}^{2 N}\right)$ for any $s \in[2, \infty)$. Thus, $R_{z}(t, z) \in L^{2}$. A standard argument shows that $z$ is also a homoclinic orbit of (HS) (see [12]). So if $z \neq 0$ is a solution of (HS), then $z$ is a homoclinic orbit of (HS).

Now, we discuss the linking structure of $\Phi$.

Lemma 2.4. Suppose $\left(R_{1}\right),\left(R_{2}\right)$ are satisfied. Then, there is a $\rho>0$ such that $\kappa:=\inf \Phi\left(\partial B_{\rho} \cap E^{+}\right)>$ 0 .

Proof. Observe that, given $\varepsilon>0$, there is $C_{\varepsilon}>0$ such that

$$
\begin{gathered}
\left|R_{z}(t, z)\right| \leq \varepsilon|z|+C_{\varepsilon}|z|^{p-1} \\
|R(t, z)| \leq \varepsilon|z|^{2}+C_{\varepsilon}|z|^{p}
\end{gathered}
$$

for all $(t, z)$, where $p \in[2, \infty)$. For $z \in E^{+}$, by Lemma 2.3 and $(2.35)$, we have

$$
\begin{aligned}
\Phi(z) & =\frac{1}{2}\|z\|^{2}-\int_{\mathbb{R}} R(t, z) \\
& \geq \frac{1}{2}\|z\|^{2}-C\left(\varepsilon\|z\|^{2}+C_{\varepsilon}\|z\|^{p}\right) .
\end{aligned}
$$

Choosing an appropriate $\varepsilon$, we see that the desired conclusion holds for some $\rho>0$.

For the asymptotically quadratic case, let $b:=\inf R_{\infty}(t)$, and we arrange all the eigenvalues (counted with multiplicity) of $A$ in $(0, b)$ by $0<\lambda_{1} \leq \lambda_{2} \leq \cdots \leq \lambda_{k}<b$ and let $e_{j}$ denote the corresponding eigenfunctions $A e_{j}=\lambda_{j} e_{j}$ for $j=1,2, \ldots, k$. Set $Y_{0}:=\operatorname{span}\left\{e_{1}, e_{2}, \ldots, e_{k}\right\}$. Note that

$$
\lambda_{1}|z|_{2}^{2} \leq\|z\|^{2} \leq \lambda_{k}|z|_{2}^{2} \quad \forall z \in Y_{0}
$$

For any subspace $W$ of $Y_{0}$, set $E_{W}=E^{-} \oplus E^{0} \oplus W$.

Lemma 2.5. Let $\left(R_{1}\right)-\left(R_{3}\right)$ be satisfied and $\rho>0$ given by Lemma 2.4. Then, for any subspace $W$ of $Y_{0}, \sup \Phi\left(E_{W}\right)<\infty$, and there is a $R_{W}>0$ such that $\sup \Phi\left(E_{W} \backslash \bar{B}_{R_{W}}\right)<\inf \Phi\left(B_{\rho} \cap E^{+}\right)$. 
Proof. It is sufficient to prove that $\Phi(z) \rightarrow-\infty$ in $E_{W}$ as $\|z\| \rightarrow \infty$. If not, then there are $M>0$ and $\left\{z_{j}\right\} \subset E_{W}$ with $\left\|z_{j}\right\| \rightarrow \infty$ such that $\Phi\left(z_{j}\right) \geq-M$ for all $j$. Denote $v_{j}:=z_{j} /\left\|z_{j}\right\|$, passing to a subsequence if necessary, $v_{j} \rightarrow v, v_{j}^{-} \rightarrow v^{-}, v_{j}^{0} \rightarrow v^{0}$, and $v_{j}^{+} \rightarrow v^{+}$. Now, we have

$$
\frac{1}{2}\left(\left\|v_{j}^{+}\right\|^{2}-\left\|v_{j}^{-}\right\|^{2}\right)-\int_{\mathbb{R}} \frac{R\left(t, z_{j}\right)}{\left\|z_{j}\right\|^{2}}=\frac{\Phi\left(z_{j}\right)}{\left\|z_{j}\right\|^{2}} \geq \frac{-M}{\left\|z_{j}\right\|^{2}},
$$

since $R(t, z) \geq 0$. If $v^{+}=0$ and $v^{0}=0$, it follows from (2.38) that

$$
\frac{1}{2}\left\|v_{j}^{-}\right\|^{2}+\int_{\mathbb{R}} \frac{R\left(t, z_{j}\right)}{\left\|z_{j}\right\|^{2}} \leq \frac{1}{2}\left\|v_{j}^{+}\right\|^{2}+\frac{M}{\left\|z_{j}\right\|^{2}} \longrightarrow 0
$$

as $j \rightarrow \infty$. Thus, we have $\left\|v_{j}^{-}\right\| \rightarrow 0$ and $\int_{\mathbb{R}}\left(R\left(t, z_{j}\right) /\left\|z_{j}\right\|^{2}\right) \rightarrow 0$, this is a contradiction with $\left\|v_{j}\right\|=1$. Therefore, there are three possibilities: (i) $v^{+} \neq 0$ and $v^{0}=0$, (ii) $v^{+}=0$ and $v^{0} \neq 0$, (iii) $v^{+} \neq 0$ and $v^{0} \neq 0$.

If (i) holds, by (2.37), one has

$$
\begin{aligned}
\left\|v^{+}\right\|^{2}-\left\|v^{-}\right\|^{2}-\int_{\mathbb{R}} R_{\infty}(t) v^{2} & \leq\left\|v^{+}\right\|^{2}-\left\|v^{-}\right\|^{2}-b|v|_{2}^{2} \\
& \leq-\left(b-\lambda_{k}\right)\left|v^{+}\right|_{2}^{2}-\left\|v^{-}\right\|^{2}-b\left|v^{-}\right|_{2}^{2-b\left|v^{0}\right|_{2}^{2}}-b\left|v^{-}\right|_{2}^{2}<0 .
\end{aligned}
$$

If (ii) or (iii) holds, similar to (i), one has

$$
\left\|v^{+}\right\|^{2}-\left\|v^{-}\right\|^{2}-\int_{\mathbb{R}} R_{\infty}(t) v^{2}<0
$$

Then, there exists $a>0$ such that

$$
\left\|v^{+}\right\|^{2}-\left\|v^{-}\right\|^{2}-\int_{-a}^{a} R_{\infty}(t) v^{2}<0
$$

Letting $\widetilde{R}(t, z):=R(t, z)-(1 / 2) R_{\infty}(t) z^{2}$, then $|\widetilde{R}(t, z)| \leq c z^{2}$ for some $c>0$ and $\widetilde{R}(t, z) / z^{2} \rightarrow 0$ as $|z| \rightarrow \infty$ uniformly in $t$. Hence, by Lebesgues dominated convergence theorem, we have

$$
\lim _{j \rightarrow \infty} \int_{-a}^{a} \frac{\tilde{R}\left(t, z_{j}\right)}{\left\|z_{j}\right\|^{2}}=\lim _{j \rightarrow \infty} \int_{-a}^{a} \frac{\tilde{R}\left(t, z_{j}\right)}{\left|z_{j}\right|^{2}}\left|v_{j}\right|^{2}=0 .
$$


Thus, (2.38)-(2.43) imply that

$$
\begin{aligned}
0 & \leq \lim _{j \rightarrow \infty}\left(\frac{1}{2}\left(\left\|v_{j}^{+}\right\|^{2}-\left\|v_{j}^{-}\right\|^{2}\right)-\int_{-a}^{a} \frac{R\left(t, z_{j}\right)}{\left\|z_{j}\right\|^{2}}\right) \\
& \leq \frac{1}{2}\left(\left\|v^{+}\right\|^{2}-\left\|v^{-}\right\|^{2}-\int_{-a}^{a} R_{\infty}(x) v^{2}\right)<0 .
\end{aligned}
$$

Now, the desired conclusion is obtained from this contradiction.

For the superquadratic case, we define $Y_{n}:=\operatorname{span}\left\{e_{1}, \ldots, e_{n}\right\}$ and $E_{n}=E^{-} \oplus E^{0} \oplus Y_{n}$, $n \in \mathbb{N}$. Then, $Y_{n}$ is a finite dimensional subspace of $E^{+}$and

$$
\lambda_{1}|z|_{2}^{2} \leq\|z\|^{2} \leq \lambda_{n}|z|_{2}^{2} \quad \forall z \in Y_{n}
$$

By $\left(\mathrm{R}_{4}\right)$, there exists $R>0$ and $\alpha>0$ such that

$$
R(t, z) \geq \alpha|z|^{2} \geq \lambda_{n}|z|^{2} \quad \forall|z| \geq R
$$

Lemma 2.6. Let $\left(R_{1}\right),\left(R_{4}\right)-\left(R_{5}\right)$ be satisfied and $\rho>0$ given by Lemma 2.4. Then, there is a sequence $\left\{R_{n}\right\}$ with $R_{n}>\rho$ such that $\sup \Phi\left(E_{n}\right)<\infty$ and $\sup \Phi\left(E_{n} \backslash \bar{B}_{n}\right)<\inf \Phi\left(B_{\rho} \cap E^{+}\right)$, where $\bar{B}_{n}=$ $\left\{z \in E_{n}:\|z\| \leq R_{n}\right\}$.

Proof. Similar to proof of Lemma 2.5. If (i) holds, by (2.45) and (2.46), it is easy to prove that

$$
\begin{aligned}
\left\|v^{+}\right\|^{2}-\left\|v^{-}\right\|^{2}-\alpha \int_{\mathbb{R}}|v|^{2} & \leq \lambda_{n}\left\|v^{+}\right\|^{2}-\left\|v^{-}\right\|^{2}-\alpha|v|_{2}^{2} \\
& =-\left(\alpha-\lambda_{n}\right)\left|v^{+}\right|_{2}^{2}-\left\|v^{-}\right\|^{2}-\alpha\left|v^{-}\right|_{2}^{2}-\alpha\left|v^{0}\right|_{2}^{2}<0 .
\end{aligned}
$$

If (ii) or (iii) holds, similar to (i), one has

$$
\left\|v^{+}\right\|^{2}-\left\|v^{-}\right\|^{2}-\alpha \int_{\mathbb{R}}|v|^{2}<0
$$

Hence, there exists $a>0$ such that

$$
\left\|v^{+}\right\|^{2}-\left\|v^{-}\right\|^{2}-\alpha \int_{-a}^{a}|v|^{2}<0
$$


Note that

$$
\begin{aligned}
\frac{\Phi\left(z_{j}\right)}{\left\|z_{j}\right\|^{2}} & \leq \frac{1}{2}\left(\left\|v_{j}^{+}\right\|^{2}-\left\|v_{j}^{-}\right\|^{2}\right)-\alpha \int_{-a}^{a} \frac{R\left(t, z_{j}\right)}{\left\|z_{j}\right\|^{2}} \\
& =\frac{1}{2}\left(\left\|v_{j}^{+}\right\|^{2}-\left\|v_{j}^{-}\right\|^{2}-\alpha \int_{-a}^{a}\left|v_{j}\right|^{2}\right)-\int_{-a}^{a} \frac{R\left(t, z_{j}\right)-(\alpha / 2)\left|z_{j}\right|^{2}}{\left\|z_{j}\right\|^{2}} \\
& \leq \frac{1}{2}\left(\left\|v_{j}^{+}\right\|^{2}-\left\|v_{j}^{-}\right\|^{2}-\alpha \int_{-a}^{a}\left|v_{j}\right|^{2}\right)+\int_{(-a, a) \cap\left\{t \in \mathbb{R}:\left|z_{j}\right| \leq R\right\}} \frac{(\alpha / 2)\left|z_{j}\right|^{2}-R\left(t, z_{j}\right)}{\left\|z_{j}\right\|^{2}} \\
& \leq \frac{1}{2}\left(\left\|v_{j}^{+}\right\|^{2}-\left\|v_{j}^{-}\right\|^{2}-\alpha \int_{-a}^{a}\left|v_{j}\right|^{2}\right)+\frac{2 a C_{R}}{\left\|z_{j}\right\|^{2}},
\end{aligned}
$$

where $C_{R}=\sup \{R(t, z): t \in(-a, a),|z| \leq R\}$. Thus, (2.38) and (2.49) imply that

$$
\begin{aligned}
0 & \leq \lim _{j \rightarrow \infty}\left(\frac{1}{2}\left(\left\|v_{j}^{+}\right\|^{2}-\left\|v_{j}^{-}\right\|^{2}\right)-\int_{-a}^{a} \frac{R\left(t, z_{j}\right)}{\left\|z_{j}\right\|^{2}}\right) \\
& \leq \frac{1}{2}\left(\left\|v^{+}\right\|^{2}-\left\|v^{-}\right\|^{2}-\alpha \int_{\Omega}|v|^{2}\right)<0,
\end{aligned}
$$

a contradiction. This proves the lemma.

As a consequence, we have the folllowing.

Lemma 2.7. Under the assumptions of Theorem 1.1 (Theorem 1.2), then letting $e \in Y_{0}\left(Y_{n}\right)$ with $\|e\|=1$, there is $R_{1}>\rho>0$, such that $\left.\Phi\right|_{\partial Q} \leq \kappa$, where $\kappa>0$ be given by Lemma $2.4, Q:=\{u=$ $\left.u^{-}+u^{0}+s e: u^{-}+u^{0} \in E^{-} \oplus E^{0}, s \geq 0,\|u\| \leq R_{1}\right\}$.

\section{The $(C)_{c}$ Condition}

In this section, we discuss the properties of the $(C)_{c}$ sequences. Recall that a sequence $\left\{z_{n}\right\} \subset$ $E$ is said to be a $(C)_{c}$ sequence if $\Phi\left(z_{n}\right) \rightarrow c$ and $\left(1+\left\|z_{n}\right\|\right) \Phi^{\prime}\left(z_{n}\right) \rightarrow 0$, $\Phi$ is said to satisfy the $(C)_{c}$ condition if any $(C)_{C}$ sequence has a convergent subsequence.

Lemma 3.1. Under the assumptions of Theorem 1.1 or Theorem 1.2, then any $(C)_{c}$-sequence is bounded.

Proof. Let $\left\{z_{j}\right\} \subset E$ be such that

$$
\Phi\left(z_{j}\right) \longrightarrow c, \quad\left(1+\left\|z_{j}\right\|\right) \Phi^{\prime}\left(z_{j}\right) \longrightarrow 0
$$

Then, there is constant $C>0$ such that we have

$$
\Phi\left(z_{j}\right)-\frac{1}{2} \Phi^{\prime}\left(z_{j}\right) z_{j}=\int_{\mathbb{R}} \widehat{R}\left(t, z_{j}\right) \leq C .
$$


Suppose to the contrary that $\left\{z_{j}\right\}$ is unbounded. Setting $v_{j}:=z_{j} /\left\|z_{j}\right\|$, then $\left\|v_{j}\right\|=1, v_{j}=$ $v_{j}^{+}+v_{j}^{0}+v_{j}^{-} \in E^{+} \oplus E^{0} \oplus E^{-}$. After passing a subsequence, we have $v_{j} \rightarrow v, v_{j}^{0} \rightarrow v^{0}$, and $\gamma=\lim _{j \rightarrow \infty}\left\|v_{j}^{+}+v_{j}^{-}\right\|^{2}$ exists. Moreover, $\left|v_{j}\right|_{s} \leq C_{s}$ for all $s \in[2, \infty)$. For $\gamma$, we have only the following two cases: $\gamma=0$ or $\gamma>0$.

First, we consider $\gamma=0$, then $\left\|v_{j}^{0}\right\|=\left|v_{j}^{0}\right|_{2} \rightarrow 1=\left|v^{0}\right|_{2}$. Denote $\tilde{z}_{j}=z_{j}^{+}+z_{j}^{-}, \widetilde{v}_{j}=v_{j}^{+}+v_{j}^{-}$. Note that by $\left(R_{3}\right)$, for any $\ell>0$, there exists a $h>0$ such that

$$
\ell \leq \widehat{R}(t, z), \quad \forall|z| \geq h
$$

For $\delta>0$, set $\Omega_{\delta}:=\left\{t \in \mathbb{R}:\left|v^{0}(t)\right| \geq 2 \delta\right\}$ and $\Omega_{j \delta}:=\left\{t \in \mathbb{R}:\left|\tilde{v}_{j}(t)\right| \geq \delta\right\}$. Since $v^{0} \in C^{1}(\mathbb{R})$ and $\left|v^{0}\right|_{2}=1,\left|\Omega_{\delta}\right|>0$ for all $\delta$ small. Moreover, we have

$$
\left|\Omega_{j \delta}\right| \leq \frac{1}{\delta^{2}} \int_{\Omega_{j \delta}}\left|\tilde{v}_{j}\right|^{2} \leq \frac{c}{\delta^{2}}\left\|\tilde{v}_{j}\right\|^{2}=\frac{c}{\delta^{2}}\left\|v_{j}^{+}+v_{j}^{+}\right\| \longrightarrow 0 \quad \text { as } j \longrightarrow \infty
$$

Hence, $\left|\Omega_{\delta} \backslash \Omega_{j \delta}\right| \rightarrow\left|\Omega_{\delta}\right|$ as $j \rightarrow \infty$. Therefore, there exists $N>0$ such that $\left|v_{j}(t)\right| \geq \delta / 2$ for all $t \in \Omega_{\delta} \backslash \Omega_{j \delta}$ with $j \geq N$; thus, $\left|z_{j}(t)\right| \geq(\delta / 2)\left\|z_{j}\right\| \geq h$ for $j \geq N$. From this and (3.3), we get

$$
\int_{\mathbb{R}} \widehat{R}\left(t, z_{j}\right) \geq \int_{\Omega_{\delta} \backslash \Omega_{j \delta}} \ell=\ell\left|\Omega_{\delta} \backslash \Omega_{j \delta}\right|
$$

contradicting (3.2) since $\ell$ is arbitrary.

Hence, $r>0$, observe that

$$
\Phi^{\prime}\left(z_{j}\right)\left(z_{j}^{+}-z_{j}^{-}\right)=\left\|z_{j}\right\|^{2}\left(\left\|\tilde{v}_{j}\right\|^{2}-\int_{\mathbb{R}} \frac{R_{z}\left(t, z_{j}\right)\left(v_{j}^{+}-v_{j}^{-}\right)\left|v_{j}\right|}{\left|z_{j}\right|}\right) .
$$

Hence,

$$
\int_{\mathbb{R}} \frac{R_{z}\left(t, z_{j}\right)\left(v_{j}^{+}-v_{j}^{-}\right)\left|v_{j}\right|}{\left|z_{j}\right|} \longrightarrow r^{2}
$$

Set

$$
g(r):=\inf \left\{\widehat{R}(t, z) \mid t \in \mathbb{R}, z \in \mathbb{R}^{2 N} \text { with }|z| \geq r\right\}
$$

for $r \geq 0$. By $\left(\mathrm{R}_{3}\right), g(r)>0$ for all $r>0$ and $g(r) \rightarrow \infty$ as $r \rightarrow \infty$. For $0 \leq a<b$, let

$$
\begin{gathered}
\Omega_{j(a, b)}:=\left\{t \in \mathbb{R}|a \leq| z_{j}(t) \mid<b\right\} \\
C_{a}^{b}:=\inf \left\{\frac{\widehat{R}(t, z)}{|z|^{2}} \mid t \in \mathbb{R}, z \in \mathbb{R}^{2 N} \text { with } a \leq|z(x)|<b\right\} .
\end{gathered}
$$


By (3.2), it is easy to prove that

$$
\left|\Omega_{j(b, \infty)}\right| \leq \frac{C}{g(b)} \longrightarrow 0
$$

as $b \rightarrow \infty$ uniformly in $t$, and, for any fixed $0<a<b$,

$$
\int_{\Omega_{j(a, b)}}\left|v_{j}\right|^{2}=\frac{1}{\left\|z_{j}\right\|^{2}} \int_{\Omega_{j(a, b)}}\left|z_{j}\right|^{2} \leq \frac{C}{C_{a}^{b}\left\|z_{j}\right\|^{2}} \longrightarrow 0
$$

as $j \rightarrow \infty$, and

$$
\int_{\Omega_{j(b, \infty)}}\left|v_{j}\right|^{s} \leq\left(\int_{\Omega_{j(b, \infty)}}\left|v_{j}\right|^{2 s}\right)^{1 / 2}\left|\Omega_{j(b, \infty)}\right|^{1 / 2} \longrightarrow 0
$$

for any $s \in[2, \infty)$, as $b \rightarrow \infty$ uniformly in $j$. Let $0<\varepsilon<\gamma^{2} / 3$. By $\left(R_{1}\right)$, there is a $a_{\varepsilon}>0$ such that

$$
\left|R_{z}(t, z)\right|<\varepsilon|z|, \quad \forall|z| \leq a_{\varepsilon} .
$$

Consequently,

$$
\int_{\Omega_{j\left(0, a_{\varepsilon}\right)}} \frac{R_{z}\left(t, z_{j}\right)\left(v_{j}^{+}-v_{j}^{-}\right)\left|v_{j}\right|}{\left|z_{j}\right|} \leq \int_{\Omega_{j\left(0, a_{\varepsilon}\right)}} \varepsilon\left|v_{j}^{+}-v_{j}^{-}\right|\left|v_{j}\right| \leq \frac{\varepsilon}{C_{2}}\left|v_{j}\right|_{2}^{2}<\varepsilon
$$

for all $j$.

By $\left(R_{2}\right)$, there is some $c>0$ such that

$$
\left|R_{z}(t, z)\right|<c|z|, \quad \forall(t, z) .
$$

By (3.11) and Hölder inequality, setting $\mu=2 v /(v-1)>2$ and $v^{\prime}=\mu / 2=v /(v-1)$, we can take large $b_{\varepsilon}$ such that

$$
\begin{aligned}
\int_{\Omega_{j\left(b b_{,}, \infty\right)}} \frac{R_{z}\left(t, z_{j}\right)\left(v_{j}^{+}-v_{j}^{-}\right)\left|v_{j}\right|}{\left|z_{j}\right|} & \leq\left(\int_{\Omega_{j(b, \infty)}} \frac{\left|R_{z}\left(t, z_{j}\right)\right|^{v}}{\left|z_{j}\right|^{v}}\right)^{1 / v}\left(\int_{\Omega_{j\left(b_{\varepsilon}, \infty\right)}}\left(\left|v_{j}^{+}-v_{j}^{-}\right|\left|v_{j}\right|\right)^{v^{\prime}}\right)^{1 / v^{\prime}} \\
& \leq c\left|\Omega_{j\left(b_{\varepsilon}, \infty\right)}\right|^{1 / v}\left(\int_{\Omega_{j\left(b_{\varepsilon}, \infty\right)}}\left|v_{j}^{+}-v_{j}^{-}\right|^{\mu}\right)^{1 / \mu}\left(\int_{\Omega_{j\left(b_{\varepsilon}, \infty\right)}}\left|v_{j}\right|^{\mu}\right)^{1 / \mu} \leq \varepsilon
\end{aligned}
$$


for all $j$. By (3.11), there is $j_{0}$ such that

$$
\int_{\Omega_{j\left(a_{\varepsilon}, b_{\varepsilon}\right)}} \frac{R_{z}\left(t, z_{j}\right)\left(v_{j}^{+}-v_{j}^{-}\right)\left|v_{j}\right|}{\left|z_{j}\right|} \leq c \int_{\Omega_{j\left(a_{\varepsilon}, b_{\varepsilon}\right)}}\left|v_{j}^{+}-v_{j}^{-}\right|\left|v_{j}\right| \leq c\left|v_{j}\right|_{2}\left(\int_{\Omega_{j\left(a_{\varepsilon}, b_{\varepsilon}\right)}}\left|v_{j}\right|^{2}\right)^{1 / 2} \leq \varepsilon
$$

for all $j \geq j_{0}$. By (3.14)-(3.17), we have

$$
\lim _{j \rightarrow \infty} \sup \int_{\mathbb{R}} \frac{R_{z}\left(t, z_{j}\right)\left(v_{j}^{+}-v_{j}^{-}\right)\left|v_{j}\right|}{\left|z_{j}\right|} \leq 3 \varepsilon<r^{2}
$$

which contradicts (3.7).

Next, we deal with the superquadratic case. Note that $2 \mu^{\prime}=2 \mu /(\mu-1)<\infty$, by (3.12), we can take large $b_{\varepsilon}>r$ such that

$$
\begin{aligned}
\int_{\Omega_{j\left(b_{\varepsilon}, \infty\right)}} & \frac{R_{z}\left(t, z_{j}\right)\left(v_{j}^{+}-v_{j}^{-}\right)\left|v_{j}\right|}{\left|z_{j}\right|} \\
\leq & \left(\int_{\Omega_{j\left(b_{\varepsilon}, \infty\right)}}\left(\frac{\left|R_{z}\left(t, z_{j}\right)\right|}{\left|z_{j}\right|}\right)^{\mu}\right)^{1 / \mu}\left(\int_{\Omega_{j\left(b_{\varepsilon}, \infty\right)}}\left(\left|v_{j}^{+}-v_{j}^{-}\right|\left|v_{j}\right|\right)^{\mu^{\prime}}\right)^{1 / \mu^{\prime}} \\
\leq & \left(\int_{\mathbb{R}} c_{1} \widehat{R}\left(t, z_{j}\right)\right)^{1 / \mu}\left(\int_{\mathbb{R}}\left|v_{j}^{+}-v_{j}^{-}\right|^{2 \mu^{\prime}}\right)^{1 / 2 \mu^{\prime}}\left(\int_{\Omega_{j\left(b_{\varepsilon, \infty}\right)}}\left|v_{j}\right|^{2 \mu^{\prime}}\right)^{1 / 2 \mu^{\prime}}<\varepsilon
\end{aligned}
$$

for all j. Clearly, (3.17) remains true. Now, the combination of (3.14), (3.17) and (3.19) implies again a contradiction with (3.7).

Let $\left\{z_{n}\right\}$ be an arbitrary $(C)_{c}$ sequence. By Lemma 3.1, it is bounded; hence, we may assume without loss of generality that $z_{n} \rightarrow z$ in $E, z_{n} \rightarrow z$ in $L^{q}$ for $q \geq 2$. Plainly, $z$ is a critical point of $\Phi$.

Lemma 3.2. Under the assumptions of Theorem 1.1 or Theorem 1.2, then $\Phi$ satisfies $(C)_{c}$ condition for all $c>0$.

Proof. Let $\left\{z_{n}\right\}$ be an arbitrary $(C)_{c}$ sequence. By (2.35), Lemmas 2.3 and 3.1, it follows from

$$
\begin{aligned}
\int_{\mathbb{R}}\left(R_{z}\left(t, z_{m}\right)-R_{z}\left(t, z_{n}\right)\right)\left(z_{m}^{+}-z_{n}^{+}\right) & \leq\left(\int_{\mathbb{R}}\left(R_{z}\left(t, z_{m}\right)-R_{z}\left(t, z_{n}\right)\right)^{2}\right)^{1 / 2}\left(\int_{\mathbb{R}}\left|z_{m}^{+}-z_{n}^{+}\right|\right)^{1 / 2} \\
& \leq c\left(\int_{\mathbb{R}}\left|z_{m}-z_{n}\right|^{2}\right)^{1 / 2} \rightarrow 0 \\
o(1) & =\left(\Phi^{\prime}\left(z_{m}\right)-\Phi^{\prime}\left(z_{n}\right), z_{m}^{+}-z_{n}^{+}\right) \\
& =\left\|z_{m}^{+}-z_{n}^{+}\right\|^{2}+\int_{\mathbb{R}}\left(R_{z}\left(t, z_{m}\right)-R_{z}\left(t, z_{n}\right)\right)\left(z_{m}^{+}-z_{n}^{+}\right) \\
& =\left\|z_{m}^{+}-z_{n}^{+}\right\|^{2}+o(1)
\end{aligned}
$$


that $z\left\|z_{m}^{+}-z_{n}^{+}\right\|^{2}=o(1)$ as $m, n \rightarrow \infty$. Similarly, we can prove that $z\left\|z_{m}^{-}-z_{n}^{-}\right\|^{2}=o(1)$ as $m, n \rightarrow \infty$. Since $\operatorname{dim} E^{0}<\infty$, thus $z_{n}^{0} \rightarrow z^{0}$. So $\left\{z_{n}\right\}$ is a Cauchy sequence in $E$. Now, the conclusion follows from the completeness of $E$.

\section{Proof of Theorems}

In this section, we give the proof for our Theorems 1.1 and 1.2. Let $E$ be a Banach space with direct sum $E=X \oplus Y$ and corresponding projections $P_{X}, P_{Y}$ onto $X, Y$. Let $\mathcal{S} \subset X^{*}$ be a dense subset, for each $s \in \mathcal{S}$, there is a seminorm on $E$ defined by

$$
p_{s}: E \longrightarrow \mathbb{R}, \quad p_{s}(z):|s(x)|+\|y\| \quad \text { for } z=x+y \in E
$$

We denote by $\tau_{\mathcal{S}}$ the topology induced by seminorm family $\left\{p_{s}\right\}, w^{*}$ denotes the weak*topology on $E^{*}$. Now, some notations are needed. For a functional $\Phi \in C^{1}(E, \mathbb{R})$, we write $\Phi_{a}=\{z \in E \mid \Phi(z) \geq a\}, \Phi^{b}=\{z \in E \mid \Phi(z) \leq b\}$, and $\Phi_{a}^{b}=\Phi_{a} \cap \Phi^{b}$. Recall that $\Phi$ is said to be weakly sequentially lower semicontinuous, if for any $z_{j} \rightarrow z$ in $E$, one has $\Phi(z) \leq$ $\liminf _{j \rightarrow \infty} \Phi\left(z_{j}\right)$, and $\Phi^{\prime}$ is said to be weakly sequentially continuous if $\lim _{j \rightarrow \infty} \Phi^{\prime}\left(z_{j}\right) w=$ $\Phi^{\prime}(z) w$ for each $w \in E$.

Suppose

$\left(\Phi_{0}\right)$ for any $c \in \mathbb{R}$, superlevel $\Phi_{c}$ is $\tau_{\mathcal{S}}$-closed, and $\Phi^{\prime}:\left(\Phi_{c}, \tau_{\mathcal{S}}\right) \rightarrow\left(E^{*}, w^{*}\right)$ is continuous;

$\left(\Phi_{1}\right)$ for any $c>0$, there exists $\xi>0$ such that $\|z\|<\xi\left\|P_{Y} z\right\|$ for all $z \in \Phi_{c}$;

$\left(\Phi_{2}\right)$ there exists $\rho>0$ such that $\kappa:=\inf \Phi\left(S_{\rho} \cap Y\right)>0$, where $S_{\rho}:=\{z \in E:\|z\|=\rho\}$;

$\left(\Phi_{3}\right)$ there exists a finite-dimensional subspace $Y_{0} \subset Y$ and $R>\rho$ such that we have for $E_{0}:=X \oplus Y_{0}$ and $B_{0}:=\left\{z \in E_{0}:\|z\| \leq R\right\}$ that $\bar{c}:=\sup \Phi\left(E_{0}\right)<\infty$ and $\sup \Phi\left(E_{0} \backslash B_{0}\right)<\inf \Phi\left(B_{\rho} \cap Y\right)$;

$\left(\Phi_{4}\right)$ there exists an increasing sequence of finite-dimensional subspaces $Y_{n} \subset Y$ and a sequence of numbers $R_{n}>\rho$ such that we have for $E_{n}:=X \oplus Y_{n}$ and $B_{n}:=\left\{z \in E_{n}\right.$ : $\left.\|z\| \leq R_{n}\right\}$ that $\bar{c}:=\sup \Phi\left(E_{n}\right)<\infty$ and $\sup \Phi\left(E_{n} \backslash B_{n}\right)<\inf \Phi\left(B_{\rho} \cap Y\right)$;

$\left(\Phi_{5}\right) \Phi$ satisfies the $(C)_{c}$ condition for $c>0$.

Now, we state three critical point theorems which will be used later (see [22]).

Theorem 4.1. Let $\Phi_{0}--\Phi_{2}$ be satisfied, and suppose there are $R>\rho>0$ and $e \in Y$ with $\|e\|=1$ such that $\sup \Phi(\partial Q) \leq \kappa$ where $Q:=\{z=x+$ te $: x \in X, t \geq 0,\|z\|<R\}$. If $\Phi$ satisfies the $(C)_{c}$ condition for all $c \leq \bar{c}$, then $\Phi$ has a critical point $u$ with $\kappa \leq \Phi(z) \leq \bar{c}$.

Theorem 4.2. Assume $\Phi$ is even and $\left(\Phi_{0}\right),\left(\Phi_{2}\right)-\left(\Phi_{3}\right)$ be satisfied. Then, $\Phi$ has at least $m:=\operatorname{dim} Y_{0}$ pairs of critical points with critical values less or equal to $\bar{c}$ provided $\Phi$ satisfies the $(C)_{c}$ condition for all $c \in[\kappa, \bar{c}]$.

Theorem 4.3. Assume $\Phi$ is even with $\Phi(0)=0$, and $\left(\Phi_{0}\right)-\left(\Phi_{2}\right),\left(\Phi_{4}\right)-\left(\Phi_{5}\right)$ be satisfied. Then, $\Phi$ has an unbounded sequence of critical values. 
Lemma 4.4. Under the assumptions of Theorem 1.1 or Theorem 1.2, then, $\Psi$ is nonnegative, weakly sequentially lower semicontinuous, and $\Psi^{\prime}$ is weakly sequentially continuous. Moreover, $\Psi^{\prime}$ is compact.

In virtue of a standard way of [23], by Lemma 2.3, it is easy to prove this lemma, so we omit it here.

Lemma 4.5. $\Phi$ satisfies $\left(\Phi_{1}\right)$.

Proof. For any $c>0$ and $u \in \Phi_{c}$, using the fact that $R(t, z) \geq 0$, one has

$$
0<c \leq \frac{1}{2}\left(\left\|z^{+}\right\|-\left\|z^{-}\right\|\right)-\int_{\mathbb{R}} R(t, z) \leq \frac{1}{2}\left\|z^{+}\right\|
$$

This yields

$$
\left\|z^{+}\right\|>\left\|z^{-}\right\|, \quad\left\|z^{+}\right\|^{2} \geq 2 c
$$

Thus it suffices to show that there exists $C>0$ such that $\left\|z^{0}\right\| \leq C\left\|z^{+}\right\|$. If it is not true, then there is a sequence $\left\{z_{j}\right\} \subset \Phi_{c}$ such that

$$
\left\|z_{j}\right\|^{2} \geq\left\|z_{j}^{0}\right\|^{2} \geq j\left\|z_{j}^{+}\right\|^{2}
$$

By (4.3), $\left\|z_{j}^{0}\right\| \rightarrow \infty$ as $j \rightarrow \infty$, hence $\left\|z_{j}\right\| \rightarrow \infty$ as $j \rightarrow \infty$. Set $v_{j}=z_{j} /\left\|z_{j}\right\|$, then $\left\|v_{j}\right\|^{2}=1$ and $\left\|v_{j}^{+}\right\|^{2} \leq 1 / j \rightarrow 0$, hence $\left\|v_{j}^{-}\right\| \rightarrow 0$ as $j \rightarrow \infty$. Therefore, we can assume there is a subsequence denoted still by $\left\{v_{j}\right\}, v_{j} \rightarrow v$, thus $v_{j} \rightarrow v^{0}$ and $\left\|v^{0}\right\|=1$. Recall that $\tilde{R}(t, z)=$ $R(t, z)-(1 / 2) R_{\infty}(t) z^{2}$, then $\tilde{R}(t, z) /|z|^{2} \rightarrow 0$ as $|z| \rightarrow \infty$ uniformly in $t$. Therefore, since $\left|z_{j}(t)\right| \rightarrow \infty$ for $v^{0}(t) \neq 0$, we have

$$
\begin{aligned}
\int_{\mathbb{R}} \frac{\tilde{R}\left(t, z_{j}\right)}{\left\|z_{j}\right\|^{2}} & \leq \int_{\mathbb{R}} \frac{\tilde{R}\left(t, z_{j}\right)\left|v_{j}-v\right|^{2}}{\left|z_{j}\right|^{2}}+\int_{\mathbb{R}} \frac{\tilde{R}\left(t, z_{j}\right)|v|^{2}}{\left|z_{j}\right|^{2}} \\
& \leq \int_{v^{0}(t) \neq 0} \frac{\tilde{R}\left(t, z_{j}\right)|v|^{2}}{\left|z_{j}\right|^{2}}+c\left|v_{j}-v\right|_{2}^{2} \longrightarrow 0 .
\end{aligned}
$$

By (4.4),

$$
\left\|z_{j}^{-}+z_{j}^{0}\right\|^{2} \geq(j-1)\left\|z_{j}^{+}\right\|^{2} \geq(j-1)\left(2 c+\left\|z_{j}^{-}\right\|^{2}+2 \int_{\mathbb{R}} R\left(t, z_{j}\right)\right)
$$

or

$$
\left\|z_{j}^{0}\right\|^{2} \geq(j-1) 2 c+(j-2)\left\|z_{j}^{-}\right\|^{2}+2(j-1) \int_{\mathbb{R}} R\left(t, z_{j}\right),
$$


which implies

$$
\begin{aligned}
\frac{1}{2(j-1)} & \geq \int_{\mathbb{R}} \frac{R\left(t, z_{j}\right)}{\left\|z_{j}\right\|^{2}}=\frac{1}{2} \int_{\mathbb{R}} R_{\infty}(t)\left|v_{j}\right|^{2}+\int_{\mathbb{R}} \frac{\tilde{R}\left(t, z_{j}\right)\left|v_{j}\right|^{2}}{\left|z_{j}\right|^{2}} \\
& \geq \frac{1}{2} b\left|v_{j}\right|_{2}^{2}+o(1),
\end{aligned}
$$

consequently, $v^{0}=0$, a contradiction.

Next, we handle the superquadratic case. Similar to the above proof, passing to a subsequence if necessary, we have $v_{j} \rightarrow v^{0}$ and $\left\|v^{0}\right\|=1$. So there exists $r>0$ such that

$$
\int_{-r}^{r}\left|v^{0}\right|^{2}>0
$$

By $\left(\mathrm{R}_{4}\right)$, we know that there exist $m>0$ and $C_{m}$ such that $R(t, z) \geq C_{m}|z|^{2}$ for $|z| \geq m$, and we have

$$
\begin{aligned}
\frac{c}{\left\|z_{j}\right\|^{2}} & \leq \frac{\Phi\left(z_{j}\right)}{\left\|z_{j}\right\|^{2}} \leq \frac{1}{2}\left(\left\|v_{j}^{+}\right\|^{2}-\left\|v_{j}^{-}\right\|^{2}\right)-C_{m} \int_{-r}^{r} \frac{R\left(t, z_{j}\right)}{\left\|z_{j}\right\|^{2}} \\
& =\frac{1}{2}\left(\left\|v_{j}^{+}\right\|^{2}-\left\|v_{j}^{-}\right\|^{2}-C_{m} \int_{-r}^{r}\left|v_{j}\right|^{2}\right)-\int_{-r}^{r} \frac{R\left(t, z_{j}\right)-\left(C_{m} / 2\right)\left|z_{j}\right|^{2}}{\left\|z_{j}\right\|^{2}} \\
& \leq \frac{1}{2}\left(\left\|v_{j}^{+}\right\|^{2}-\left\|v_{j}^{-}\right\|^{2}-C_{m} \int_{-r}^{r}\left|v_{j}\right|^{2}\right)+\int_{(-r, r) \cap\left\{t \in \mathbb{R}:\left|z_{j}\right| \leq m\right\}} \frac{\left(C_{m} / 2\right)\left|z_{j}\right|^{2}-R\left(t, z_{j}\right)}{\left\|z_{j}\right\|^{2}} \\
& \leq \frac{1}{2}\left(\left\|v_{j}^{+}\right\|^{2}-\left\|v_{j}^{-}\right\|^{2}-C_{m} \int_{-r}^{r}\left|v_{j}\right|^{2}\right)+\frac{2 r C_{m}}{\left\|z_{j}\right\|^{2}} .
\end{aligned}
$$

Then,

$$
\begin{aligned}
0 & \leq \lim _{j \rightarrow \infty}\left(\frac{1}{2}\left(\left\|v_{j}^{+}\right\|^{2}-\left\|v_{j}^{-}\right\|^{2}\right)-\int_{-r}^{r} \frac{R\left(t, z_{j}\right)}{\left\|z_{j}\right\|^{2}}\right) \\
& \leq-C_{m} \int_{-r}^{r}\left|v^{0}\right|^{2}<0,
\end{aligned}
$$

a contradiction.

\section{Proof of Theorem 1.1. Existence of a Least Energy Solution}

With $X=E^{-} \oplus E^{0}$ and $Y=E^{+}$, the condition $\left(\Phi_{0}\right)$ holds by Lemma 4.4 and $\left(\Phi_{1}\right)$ holds by Lemma 4.5. Lemma 2.4 implies $\left(\Phi_{2}\right)$. Lemma 2.7 shows that $\Phi$ possesses the linking structure of Theorem 4.1, and Lemma 3.2 implies $\Phi$ satisfies $\left(\Phi_{5}\right)$. Therefore, $\Phi$ has at least one critical point $z$ with $\Phi(z) \geq \mathcal{K}>0$. Let $\mathcal{K}=\left\{z \in E \backslash\{0\}: \Phi^{\prime}(z)=0\right\}$ be the set of nontrivial points of $\Phi$. 
Then, $\mathcal{K} \neq \emptyset$. We claim that $\theta=\inf \{\Phi(z) \mid z \in \mathcal{K}\}$ is achieved. Let $\left\{z_{j}\right\} \subset \mathcal{K}$ be a minimizing sequence for $\theta$, then $\left\{z_{j}\right\}$ is bounded by Lemma 3.1. By Lemma 3.2, there exists a renamed subsequence such that $z_{j} \rightarrow z$, and

$$
\theta=\lim _{j \rightarrow \infty} \Phi\left(z_{j}\right)=\Phi(z)
$$

from which it follows that $\theta$ is achieved.

\section{Multiplicity}

$\Phi$ is even provided $R(t, z)$ is even in $z$. Lemma 2.5 says that $\Phi$ satisfies $\left(\Phi_{3}\right)$ with $\operatorname{dim} Y_{0}=k$. Therefore, $\Phi$ has at least $k$ pairs of nontrivial critical points by Theorem 4.2.

Proof of Theorem 1.2. Existence of a Least Energy Solution

Repeating the above proof, we know that $\theta$ is achieved by some nonzero critical point.

\section{Existence of Infinitely Many Solutions}

$\Phi$ is even provided $R(t, z)$ is even in $z$. Lemma 2.6 says that $\Phi$ satisfies $\left(\Phi_{4}\right)$. Therefore, $\Phi$ has an unbounded critical values by Theorem 4.3, and hence the equation (HS) has infinitely many solutions.

\section{Acknowledgments}

This work is supported partly by NSFC (11061040), NSFY of Yunnan Province (2008CD112 and 2009CD043), and the Foundation of Education Committee of Yunnan Province, China.

\section{References}

[1] V. Coti-Zelati and P. H. Rabinowitz, "Homoclinic orbits for second order Hamiltonian systems possessing superquadratic potentials," Journal of the American Mathematical Society, vol. 4, no. 4, pp. 693-727, 1991.

[2] Y. H. Ding, "Existence and multiplicity results for homoclinic solutions to a class of Hamiltonian systems," Nonlinear Analysis. Theory, Methods \& Applications, vol. 25, no. 11, pp. 1095-1113, 1995.

[3] Y. H. Ding and M. Girardi, "Periodic and homoclinic solutions to a class of Hamiltonian systems with the potentials changing sign," Dynamic Systems and Applications, vol. 2, no. 1, pp. 131-145, 1993.

[4] W. Omana and M. Willem, "Homoclinic orbits for a class of Hamiltonian systems," Differential and Integral Equations, vol. 5, no. 5, pp. 1115-1120, 1992.

[5] P. H. Rabinowitz, "Homoclinic orbits for a class of Hamiltonian systems," Proceedings of the Royal Society of Edinburgh, vol. 114, no. 1-2, pp. 33-38, 1990.

[6] V. Coti-Zelati, I. Ekeland, and . Séré, "A variational approach to homoclinic orbits in Hamiltonian systems," Mathematische Annalen, vol. 288, no. 1, pp. 133-160, 1990.

[7] Y. H. Ding, "Multiple homoclinics in a Hamiltonian system with asymptotically or super linear terms," Communications in Contemporary Mathematics, vol. 8, no. 4, pp. 453-480, 2006.

[8] Y. H. Ding and M. Girardi, "Infinitely many homoclinic orbits of a Hamiltonian system with symmetry," Nonlinear Analysis. Theory, Methods E Applications, vol. 38, no. 3, pp. 391-415, 1999.

[9] Y. H. Ding and L. Jeanjean, "Homoclinic orbits for a nonperiodic Hamiltonian system," Journal of Differential Equations, vol. 237, no. 2, pp. 473-490, 2007. 
[10] Y. H. Ding and S. J. Li, "Homoclinic orbits for first order Hamiltonian systems," Journal of Mathematical Analysis and Applications, vol. 189, no. 2, pp. 585-601, 1995.

[11] Y. H. Ding and C. Lee, "Existence and exponential decay of homoclinics in a nonperiodic superquadratic Hamiltonian system," Journal of Differential Equations, vol. 246, no. 7, pp. 2829-2848, 2009.

[12] Y. H. Ding and M. Willem, "Homoclinic orbits of a Hamiltonian system," Journal of Applied Mathematics and Physics, vol. 50, no. 5, pp. 759-778, 1999.

[13] H. Hofer and K. Wysocki, "First order elliptic systems and the existence of homoclinic orbits in Hamiltonian systems," Mathematische Annalen, vol. 288, no. 3, pp. 483-503, 1990.

[14] P. H. Rabinowitz and K. Tanaka, "Some results on connecting orbits for a class of Hamiltonian systems," Mathematische Zeitschrift, vol. 206, no. 3, pp. 473-499, 1991.

[15] E. Séré, "Existence of infinitely many homoclinic orbits in Hamiltonian systems," Mathematische Zeitschrift, vol. 209, no. 1, pp. 27-42, 1992.

[16] A. Szulkin and W. Zou, "Homoclinic orbits for asymptotically linear Hamiltonian systems," Journal of Functional Analysis, vol. 187, no. 1, pp. 25-41, 2001.

[17] K. Tanaka, "Homoclinic orbits in a first order superquadratic Hamiltonian system: convergence of subharmonic orbits," Journal of Differential Equations, vol. 94, no. 2, pp. 315-339, 1991.

[18] J. Wang, J. Xu, and F. Zhang, "Homoclinic orbits for superlinear Hamiltonian systems without Ambrosetti-Rabinowitz growth condition," Discrete and Continuous Dynamical Systems, vol. 27, no. 3, pp. 1241-1257, 2010.

[19] Y. H. Ding and B. Ruf, "Solutions of a nonlinear Dirac equation with external fields," Archive for Rational Mechanics and Analysis, vol. 190, no. 1, pp. 57-82, 2008.

[20] H. Triebel, Interpolation Theory, Function Spaces, Differential Operators, vol. 18, North-Holland Publishing, Amsterdam, Holland, 1978.

[21] D. E. Edmunds and W. D. Evans, Spectral Theory and Differential Operators, Clarendon Press, Oxford, UK, 1987.

[22] T. Bartsch and Y. Ding, "Deformation theorems on non-metrizable vector spaces and applications to critical point theory," Mathematische Nachrichten, vol. 279, no. 12, pp. 1267-1288, 2006.

[23] M. Willem, Minimax Theorems, Birkhäuser, Berlin, Germany, 1996. 


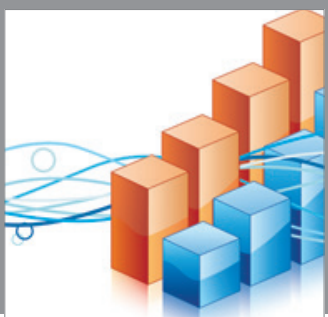

Advances in

Operations Research

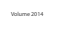

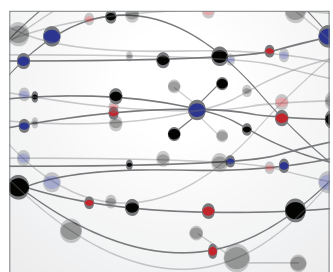

\section{The Scientific} World Journal
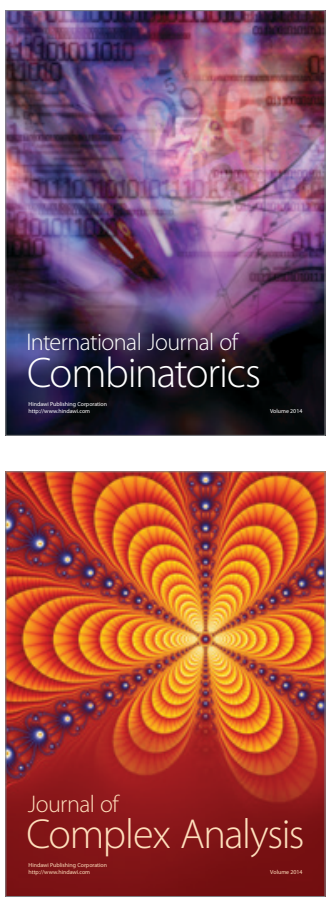

International Journal of

Mathematics and

Mathematical

Sciences
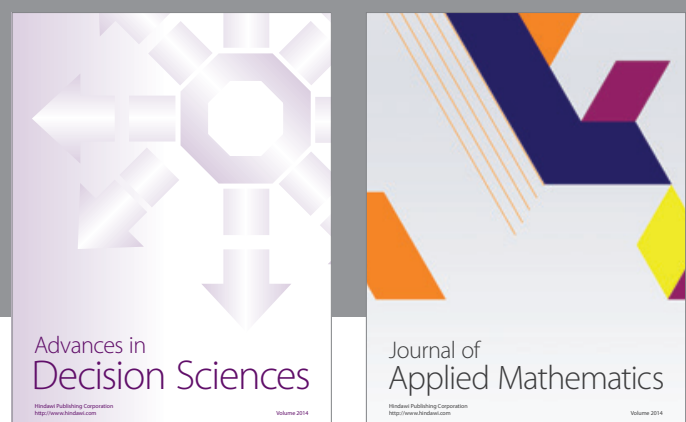

Journal of

Applied Mathematics
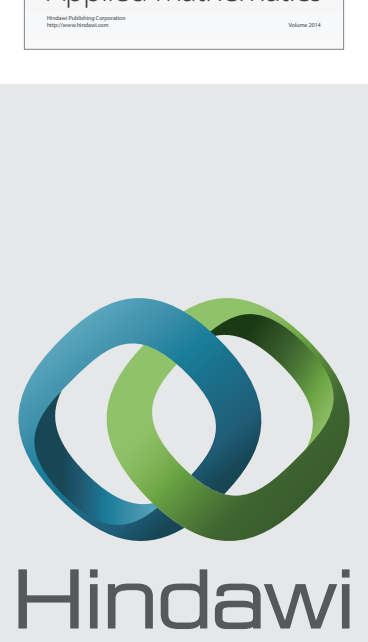

Submit your manuscripts at http://www.hindawi.com
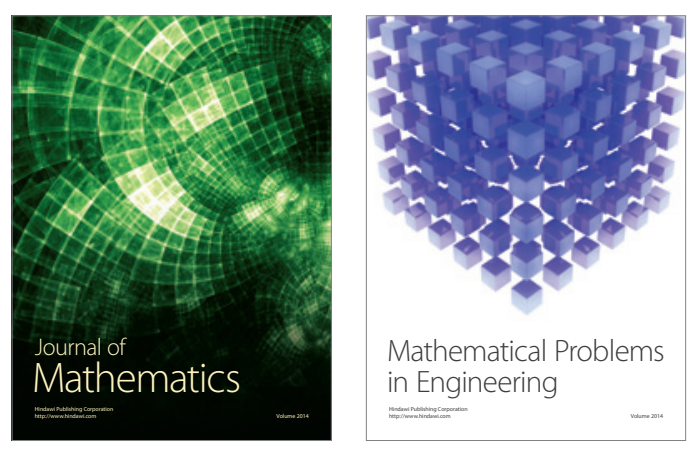

Mathematical Problems in Engineering
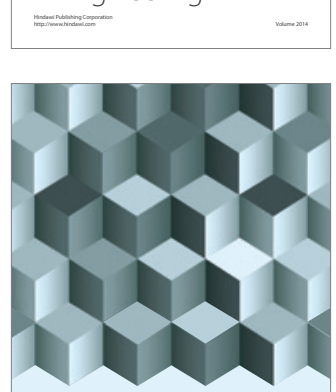

Journal of

Function Spaces
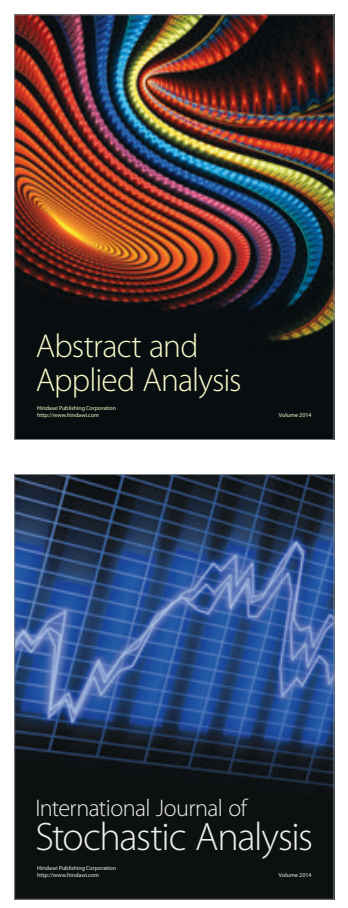

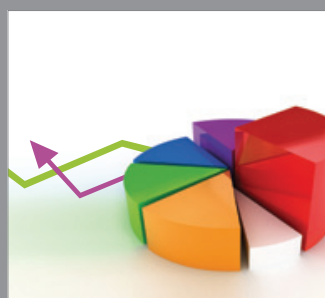

ournal of

Probability and Statistics

Promensencen
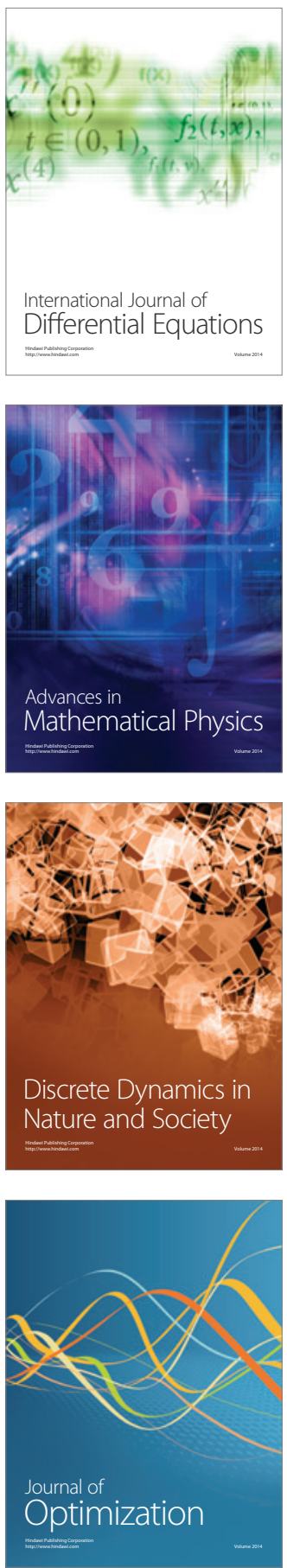